\title{
Partager un corpus vidéo dans la recherche en éducation : analyses et regards pluriels dans le cadre du projet ViSA
}

Andrée Tiberghien, Marie-Pierre Chopin, Laurent Lima, Laurent Talbot, Aline Arrieu-Mutel et Abdelkarim Zaid

\section{CpenEdition}

\section{Journals}

Édition électronique

URL : http://journals.openedition.org/educationdidactique/1686

DOI : 10.4000/educationdidactique.1686

ISSN : 2111-4838

Éditeur

Presses universitaires de Rennes

Édition imprimée

Date de publication : 30 novembre 2012

Pagination : 9-17

ISBN : 978-2-7535-2254-1

ISSN : 1956-3485

Référence électronique

Andrée Tiberghien, Marie-Pierre Chopin, Laurent Lima, Laurent Talbot, Aline Arrieu-Mutel et Abdelkarim Zaid, «Partager un corpus vidéo dans la recherche en éducation : analyses et regards pluriels dans le cadre du projet ViSA », Éducation et didactique [En ligne], 6-3 | novembre 2012, mis en ligne le 30 novembre 2014, consulté le 08 décembre 2020. URL : http://journals.openedition.org/ educationdidactique/1686 ; DOI : https://doi.org/10.4000/educationdidactique.1686 


\title{
PARTAGER UN CORPUS VIDÉO DANS LA RECHERCHE EN ÉDUCATION : ANALYSES ET REGARDS PLURIELS DANS LE CADRE DU PROJET VISA
}

\author{
Andrée Tiberghien (ICAR, CNRS-Université de Lyon) \\ Marie-Pierre Chopin (LACES-ADS, Université Bordeaux Segalen) \\ Laurent Lima (LSE, Université PMF Grenoble) \\ Laurent Talbot \& Aline Arieu-Mutel () \\ Abdelkarim Zaid (Theodile, Université Lille 2)
}

Andrée Tiberghien et al.

\section{Introduction du numéro}

Ce numéro spécial présente un travail coordonné de chercheurs en éducation scolaire qui étudient les pratiques d'enseignement-apprentissage en classe. Il a été mené dans le cadre de la structure fédérative de recherche ViSA ${ }^{1}$ (Video de Situations d'enseignement et d'Apprentissage). Cette structure qui réunit onze unités de recherche vise à :

- développer une réflexion épistémologique sur la nature des données vidéo;

- systématiser les pratiques de constitution et d'utilisation de données vidéo;

- outiller et former les chercheurs afin de ne pas perdre de temps à résoudre des problèmes déjà traités par d'autres;

- reconfigurer des pratiques de recherche plus collectives, ouvrant sur des études de plus grande ampleur et plus solidement étayées, en particulier dans une perspective de renouvellement théorique.

Ce travail sur un même corpus est un exemple de pratiques collectives de recherche qui va nourrir une réflexion épistémologique et heuristique ainsi que la propre formation des enseignants-chercheurs. Dans cette perspective, cinq chercheurs ont étudié un même corpus vidéo comportant l'enregistrement d'une séance d'enseignement et des documents associés. De telles études visent à approfondir notre compréhension des phénomènes d'enseignement et d'apprentissage qui ont lieu en classe, de repérer des dynamiques de pratiques en situation, d'identifier des processus organisateurs de ces dynamiques qui constituent autant de variations et de stabilités. Il s'agit d'un enjeu majeur de la recherche en éducation aussi bien pour la formation des maîtres que pour l'organisation de nos systèmes éducatifs, même si bien sûr d'autres types d'études sont nécessaires. La vidéo permet d'approcher la classe avec des méthodologies renouvelées (Veillard et Tiberghien, à paraître, Tiberghien et Sensevy sous presse, Sensevy, 2011; Talbot, 2008). Ainsi voir et revoir une vidéo permet de construire et de tester des hypothèses (Engle, R. A., Conant, F. R., \& Greeno, J. G, 2007), et de confronter des analyses. C'est ce que voudrait montrer ce numéro spécial. L'enjeu du travail présenté était de réaliser une étude d'un même corpus avec des perspectives théoriques différentes afin de rendre explicite la variété des approches et le type de résultat qu'elles permettent d'obtenir. Il s'agit d'une étape importante dans l'approfondissement des analyses de classe.

Dans cette introduction, nous présentons tout d'abord la genèse et les objectifs de cette recherche, puis nous donnons dans un second temps des informations relatives au corpus commun sur lequel a porté ce travail collectif.

\section{Lorigine du travail collectif}

Depuis plusieurs années, une communauté de chercheurs s'est formée autour de la question de l'analyse de vidéos de situations ayant un enjeu d'apprentissage dans le cadre du projet ViSA. La base de données réalisée et encore en développement rend possible la mise à disposition de vidéos et de leurs documents associés à d'autres chercheurs que ceux à l'origine de la collecte. Plus largement, il s'agit d'approfondir l'analyse de données comportant des vidéos de situation d'enseignement-apprentissage de l'école maternelle à l'enseignement supérieur ainsi qu'en situations de travail et informelles. Dans 
cette perspective, le conseil scientifique de ViSA a décidé de lancer une activité collective de plusieurs analyses d'un même corpus et d'en faire la présentation et l'analyse lors des rencontres régulières des « journées ViSA ». Ainsi cinq chercheurs ont joué ce jeu pour une présentation lors des journées des 13 et 14 octobre 2011 qui se sont terminées par une table ronde autour de cette étude.

Ce travail collectif n'aurait pas pu se réaliser sans un parcours préalable commun avec cette communauté-car l'entreprise comporte des risques. Comme l'explique Wendy Leeds-Hurtwitz, participante à la table ronde (dans ce volume), cela peut être difficile par exemple de risquer de s'apercevoir que l'on n'a pas vu quelque chose de critique dans les données analysées alors que d'autres l'ont pris en compte tout naturellement. En revanche l'enjeu d'ouverture, d'explicitation des cadres théoriques et des méthodologies que permet la confrontation des analyses est suffisamment motivant pour se lancer. De même, la perspective de construire des regards complémentaires dans la compréhension et l'explication des pratiques motive de tels travaux collaboratifs.

C'est ainsi que les cinq chercheurs se sont mis d'accord après quelques échanges pour travailler sur une vidéo d'une séance de classe.

\section{L'historique}

Les principales étapes de cette collaboration sont les suivantes.

La sélection du corpus était essentielle pour les cinq chercheurs. Ceux-ci se sont mis d'accord sur un enregistrement vidéo ${ }^{2}$. Cet enregistrement vidéo a été sélectionné avec les documents associés et la possibilité de compléter les informations pour certains des cinq chercheurs (voir l'article de Laurent Talbot). En effet, il s'agit de l'enregistrement d'une séance de classe qui s'est tenue le 8 octobre 2010. Le fait que le démarrage du travail ait eu lieu la même année académique que celle de la prise vidéo a permis à certains de compléter les données. Il faut noter que cet enregistrement n'avait pas encore été analysé par le groupe de projet qui avait réalisé la récolte de ces données; un des cinq chercheurs participait à ce groupe (Andrée Tiberghien).
Ceci nous amène à donner quelques points de repère sur l'historique du travail.

Le choix de la vidéo et l'analyse ont été rythmés par plusieurs discussions:

- deux discussions à distance (via Internet) ont permis de choisir la vidéo (février et mars 2011);

- après une première discussion à distance sur les analyses (avril 2011), des échanges d'informations sur les données ainsi que de la transcription de la séance ont eu lieu;

- une rencontre s'est tenue en juin 2011 à Lyon où l'échange a porté sur les approches théoriques choisies pour l'analyse;

- enfin un dernier échange a eu lieu en septembre 2011 avant l'envoi par chacun d'un texte aux participants de la table ronde.

Une dernière remarque doit être faite sur la construction de nos analyses: les cinq chercheurs avaient une pratique de l'analyse de classe, mais certains avaient une plus longue expérience de l'analyse de vidéo. Cependant, tous, ont été conduits à adapter leurs questions de recherche au corpus. Ainsi, la récolte du corpus ne s'est pas déroulée ultérieurement au cadrage théorique comme c'est le cas habituellement mais préalablement aux questions de recherche. En revanche, tous les chercheurs ont adapté un cadre théorique déjà élaboré. Ceci a été possible car la problématique globale de mieux comprendre et expliquer les pratiques d'enseignement-apprentissage est partagée.

Les journées ViSA ont permis de présenter ces études et d'en débattre au cours d'une table ronde.

\section{Les différents articles et commentaires}

Ce numéro présente les cinq recherches et les commentaires des participants à cette table ronde.

Dans le premier article, Aline Arrieu-Mutel et Laurent Talbot tentent de décrire, comprendre et expliquer les pratiques d'enseignement du professeur en se centrant principalement sur son activité 
verbale et plus particulièrement sur ses pratiques évaluatives orales durant la séance. Le cadre conceptuel convoqué dans un premier temps est celui de l'approche sociocognitive d'Albert Bandura.

Abdelkarim Zaid analyse l'évolution de l'action de l'enseignant en tant qu'action sur les performances didactiques des élèves, dans la situation d'enseignement et d'apprentissage étudiée La notion de performance didactique est brièvement présentée et un dispositif méthodologique l'opérationnalisant est détaillé. Les résultats mettent en évidence qu'au sein des contraintes de la situation, l'action de l'enseignant prend en compte les performances des élèves et traduit de manière constante les caractéristiques d'un processus de modélisation, une dynamique entre référent empirique et modèle construit.

Marie-Pierre Chopin convoque le modèle de l'hétérogénéisation didactique, en association avec les recherches sur la mémoire didactique, pour mettre en évidence la dimension spiralaire du temps de la diffusion des savoirs dans la classe. Elle montre en quoi, alors que l'unité vidéo laisse d'abord apparaître une impression de dysfonctionnement de la leçon (tout a l'air de se passer comme si le temps didactique n'avançait pas), professeur et élèves concourent en réalité activement à la diffusion de la connaissance, par des moyens différents. De cette différence, dont elle propose de comprendre l'origine dans une perspective anthropologique, découle la difficulté à construire une temporalité commune (une sorte d'idiorythmie au plein sens de Barthes) entre l'enseignant et ses élèves.

Laurent Lima s'intéresse à ce que pourraient comprendre les élèves du discours de l'enseignant. Pour cela, il adapte des modèles d'analyse créés pour étudier la compréhension de l'écrit à l'étude du discours de l'enseignant considéré comme un récit dont une partie n'est interprétable qu'en référence à la situation d'énonciation. Il produit une analyse des référents introduits dans le discours en se fondant sur le concept d'activation qui lui permet d'une part d'identifier les thèmes du discours et d'autre part d'étudier l'évolution temporelle de ces thèmes. À partir de cette première analyse thématique qui conduit à identifier une structure en 6 épisodes, une analyse détaillée de la structure référentielle et de la temporalité d'apparition des référents dans chaque épisode au sein de cette structure le conduit à proposer un modèle de représentation du discours de l'enseignant, ainsi que des pistes de connaissances pouvant être acquises par les élèves

Andrée Tiberghien analyse la séance à partir de la théorie de l'action conjointe en travaillant à deux échelles, une échelle mésoscopique et une échelle microscopique pour rendre compte de la dynamique de l'évolution du savoir dans la classe. Une analyse en termes de jeux didactiques vise à rendre compte de l'évolution du sens pris par le savoir, au sein de la classe, dans les transactions entre professeurs et élèves. Cette analyse conduit à considérer que cette séance ${ }^{3}$ permet en grande partie au professeur et aux élèves de se recaler dans la mesure où le professeur et les élèves s'emploient à partager les mêmes supports (spectres, schéma, etc.) et une signification commune des activités réalisées la séance précédente.

Ces deux derniers textes offrent chacun une analyse thématique de la séance qui sont comparées succinctement dans une annexe commune.

L'ordre de présentation de ces textes n'est pas essentiel, le lecteur pourra choisir selon ses intérêts. Nous avons toutefois opté pour une organisation des contributions allant de l'analyse la plus globale de la classe à celle la plus centrée sur le savoir en jeu.

Des mises en perspective de ces cinq textes sont proposées par Serge Leblanc, Wendy Leeds-Hurwitz, Bernard Sarrazy, Gérard Sensevy et Patrice Venturini. Elles prennent du recul sur les travaux empiriques d'analyse de classe à partir de données comprenant des vidéos et ainsi elles contribuent à la compréhension des cinq approches et en proposent des structurations nouvelles.

\section{Présentation de l'enregistrement vidéo étudié par les cinq chercheurs de ViSA}

L'enregistrement choisi a été réalisé le 8 octobre 2010 dans une classe de seconde d'un lycée de centre-ville pendant une séance d'enseignement de physique. Il s'agit de la huitième séance de l'enseignement de physique et chimie de l'année académique.

La durée de cet enseignement est programmée hebdomadairement: chaque semaine, il y a d'une part 
une séance de 1 h 30 en demi-classe pour les travaux pratiques (TP). Le professeur répète donc deux fois la même séance à deux sous-groupes d'élèves. D'autre part, l'enseignement en classe entière est de 1 h 30 par semaine qui, dans ce lycée, fonctionne sur un rythme de deux semaines: une semaine sur deux il est de $1 \mathrm{~h}$ ou de $2 \mathrm{~h}$. La séance étudiée se situe dans ce dernier cas où la durée totale était de 2 heures, seule la première heure a été étudiée.

Nous donnons succinctement quelques informations sur l'enseignement puis sur la problématique générale de la recherche à l'origine de l'enregistrement.

\section{Contexte de l'enseignement}

L'année académique 2010-2011 est la première année d'un nouveau programme (voir en annexe des extraits du contenu de ce programme). Ce nouveau programme est structuré en thèmes au nombre de trois: Univers, Santé et Sport. Il faut noter que deux de ces thèmes ne sont pas liés à la physique ou la chimie mais à des questions sociales. Ceci est tout à fait nouveau et les auteurs du programme justifient ce choix en considérant que l'approche thématique permet de développer l'intérêt pour les sciences.

Le professeur a commencé l'année par le thème « Univers » du programme de physique. Les séances 7 et 8 portent sur la deuxième partie de ce thème du programme officiel:

« Les étoiles: l'analyse de la lumière provenant des étoiles donne des informations sur leur température et leur composition. Cette analyse nécessite l'utilisation de systèmes dispersifs. » (MEN, 2010 (BOEN)).

Ce programme associe à ce thème des compétences très variées comme:

- Savoir qu'un corps chaud émet un rayonnement continu, dont les propriétés dépendent de la température.

- Repérer, par sa longueur d'onde dans un spectre d'émission ou d'absorption, une radiation caractéristique d'une entité chimique.
- Utiliser un système dispersif pour visualiser des spectres d'émission et d'absorption et comparer ces spectres à celui de la lumière blanche.

- Savoir que la longueur d'onde caractérise dans l'air et dans le vide une radiation monochromatique. Interpréter le spectre de la lumière émise par une étoile: température de surface et entités chimiques présentes dans l'atmosphère de l'étoile.

- Connaître la composition chimique du Soleil. (MEN, 2010)

Dans cette partie du programme, les notions comme celles de rayonnement continu, température de surface, atmosphère d'une étoile, sont inconnues de la grande majorité des élèves. De même, ils rencontrent pour la première fois la notion de spectres d'émission et d'absorption qui est au cœur de la séance qui sera analysée dans ce numéro thématique. Le programme demande donc d'introduire ces notions dans le cadre du thème de l'univers et plus spécifiquement des étoiles avec comme enjeu la compréhension des phénomènes du point de vue de la physique.

\section{Séquence conçue et utilisée par l'enseignant observé}

Le professeur utilise une séquence qui a été conçue en groupe ${ }^{4}$ à la fin de l'année académique précédente. Mais le programme étant paru très tard, la conception de cette séquence n’a pas été très travaillée.

La séquence conçue sur le thème univers comporte quatre chapitres:

- Chapitre 1: "Science et univers " qui introduit à l'univers mais aussi au fonctionnement des sciences (questions scientifique et non scientifique).

- Chapitre 2: «Outils de description de l'univers » (ordre de grandeur, maquette du système solaire, vitesse de la lumière et année de lumière).

- Chapitre 3: « Spectres et phénomène de dispersion $»$. Ce chapitre comprend 
-une activité d'introduction sur ce que nous apprend la lumière émise par les étoiles avec le document 1 dont il sera question pendant la séance 8 donnant les spectres de 6 étoiles plus ou moins chaudes

- l'activité 1: Se donner les moyens d'étudier le phénomène de dispersion;

- l'activité 2: Dans la peau de Newton: une prévision sur le rôle du prisme.

- Chapitre 4: «Spectres d'émission et d'absorption; analyse spectrale ». Ce chapitre comprend:

- l'activité 1: Premières utilisations d'un spectroscope;

- l'activité 2: Lumière émise par un corps chauffé Partie A - Étude du spectre de la lumière émise par une ampoule à incandescence et Partie B - Une interprétation de la couleur des étoiles;

- l'activité 3: Spectres d'émission de différentes lampes.;

- l'activité 4: Des spectres qui semblent incomplets.;

- l'activité 5 : Détermination de la composition d'une étoile à l'aide de son spectre.

Les séances 7 et 8 , c'est-à-dire la séance précédant la séance étudiée et la séance étudiée traitent du chapitre 4. Pour situer la séance 8 , il est important de la présenter en lien avec la séance 7 . En effet lors de la séance 7 les élèves ont réalisé plusieurs activités expérimentales qui sont reprises dans la séance 8 . La séance 7 correspond à une séance de TP au sens où les élèves travaillent en demi-classe (le professeur fait deux fois cette séance) et sa durée est de 1 h 30. La séance 8 est en classe entière.

\section{La classe et la disposition des caméras}

Cette classe compte 36 élèves. Le plan de la classe est donné dans la figure 1 (page suivante). À noter que le professeur n'a pas la place de se déplacer entre chacun des rangs.

\section{Contexte de la recherche}

La problématique générale à l'origine du recueil de données porte sur l'établissement du contrat didactique dans la situation d'enseignement-apprentissage. Pour cela, il a été décidé de filmer les classes dès la première séance, c'est-à-dire quand le professeur prend contact avec ses élèves pour la toute première fois.

6 professeurs ont été filmés dans 3 classes de trois établissements différents; dans chaque établissement, le professeur de physique-chimie et le professeur de Sciences de la Vie et de la Terre (SVT) de la même classe ont été filmés. Toutes les séances du début de l'année jusqu'à la mi ou fin octobre ont été enregistrées.

La séance choisie se situe en classe entière à un moment où le professeur et les élèves commencent à se connaître: c'est-à-dire que le professeur perçoit peu à peu ce qu'il peut attendre des élèves. Inversement, les élèves commencent à savoir ce qu'ils peuvent attendre du professeur.

Cette problématique n'a pas été reprise dans les analyses présentées pour les journées ViSA. Une telle démarche nécessite une analyse sur une séquence (série de séances). 
PARTAGER UN CORPUS VIDÉO DANS LA RECHERCHE EN ÉDUCATION...

Andrée Tiberghien \& al

Bureau prof

\begin{tabular}{|l|l|l|l|}
\hline $\mathrm{F}$ & $\mathrm{F}$ & $\mathrm{F}$ & $\mathrm{F}$ \\
29 & 34 & $16(\mathrm{D})$ & $3(\mathrm{D})$ \\
B1 B2 & M1 F2 & M1 M2 & M1 B2 \\
\hline
\end{tabular}

\begin{tabular}{|c|c|c|c|c|c|c|c|c|}
\hline Caméra élève & $\begin{array}{l}\text { G } \\
35 \\
\text { M1 B2 }\end{array}$ & $\begin{array}{l}G \\
31(\mathrm{D}) \\
\text { F1 M2 }\end{array}$ & $\begin{array}{l}\mathrm{F} \\
32 \\
\text { B1 B2 }\end{array}$ & $\begin{array}{l}F \\
30 \\
\text { M1 82 }\end{array}$ & $\begin{array}{l}F \\
7 \\
\text { M1 B2 }\end{array}$ & $\begin{array}{l}F \\
23 \\
F 1 M 2\end{array}$ & $\begin{array}{l}\text { F } \\
4 \\
\text { B1 B2 }\end{array}$ & $\begin{array}{l}F \\
14 \\
\text { M1 M2 }\end{array}$ \\
\hline
\end{tabular}

\begin{tabular}{|l|l|l|l|l|l|l|l|}
\hline G1 & G2 & G & G & F & G & G & F \\
B1 B2 & Filmé & 24 & 25 & 18 & 15 & 20 & 9 \\
& $\begin{array}{l}\text { M1 M2 (ou } \\
\text { B2...) }\end{array}$ & B1B2 & B1B2 & F1 M2 & F1 M2 & M1 F2 & B1 B2 \\
\cline { 2 - 6 } & & & & & & \\
\hline
\end{tabular}

\begin{tabular}{|l|l|l|l|l|l|l|l|}
\hline G & G & G & G & G & G & G & F \\
B6 & 11 & 12 & 19 & 5 (D) & 13 (D) & 17 (D) & $28(D)$ \\
& M1 M2 & B1 B2 & F1 F2 & F1 M2 & B1 B2 & M1 M2 & M1 F2 \\
\hline
\end{tabular}

\begin{tabular}{|l|l|l|l|l|l|l|l|}
\hline F & F & F & F & G & G & G & G \\
6 & 26 & 21 & 8 & 10 & 27 & 33 & 22 \\
B1 B2 & F1 F2 & M1 B2 & F1 F2 & M1 B2 & F1 F2 & B1 B2 & B1 B2 \\
\hline
\end{tabular}

Figure 1: plan de la classe Code: $G=$ Garcon F=Fille

Pour chaque élève:

Son numéro d'identification (anonymisation)

Son niveau donné par l'enseignant (premières notes et comportement général en classe) au moment de la séance:

B1=Bon, M1=Moyen, F1: Faible

Et au mois de mai 2011 (soit 9 mois après la séance vidéoscopée) : B2=Bon, M2: Moyen, F2: Faible, (D): Doublants 


\section{NOTES}

1. Voir le site: http://visa.inrp.fr

2. On pourra remarquer l'influence possible, dans ce choix, des cadres théoriques familièrement sollicité par les chercheurs, ainsi que le cadrage du document vidéo (plan large, vue prof et tableau)

3. Cette séance porte sur les spectres d'émission et d'absorption et l'analyse spectrale

4. Il s'agit d'un groupe de recherche développement SESAMES regroupant des enseignants et des chercheurs de l'UMR ICAR qui est financé pour les enseignants par l'Institut Français d'Éducation (ex INRP). Les travaux de ce groupe sont mis sur le site pegase. inrp. fr

\section{RÉFÉRENCES}

Engle, R. A., Conant, F. R., \& Greeno, J. G. (2007). Progressive refinement of hypotheses in video-supported research. In R. Goldman, R. Pea, B. Barron \& S. J. Derry (Eds.), Video research in the learning sciences (p. 239-254). Mawah, New Jersey: Lawrence Erlbaum Associates.

MEN (ministère de l'Éducation nationale). (2010). Programmes d'enseignement de la classe de seconde. Bulletin officiel, 4 ( $\mathrm{n}^{\circ} 4$ du 29 avril 2010 Bulletin officiel spécial).

Tiberghien, A., \& Sensevy, G. (sous presse). Video studies: Time and duration in the teaching-learning processes. In J. Dillon \& D. Jorde (Eds.), Handbook "The World of Science Education” volume 4. Rotterdam/ Boston/Taipei : Sense Publisers.

Sensevy, G. (2011). Le sens du savoir. Éléments pour une théorie de l'action conjointe en didactique. Bruxelles: de Boeck.

Talbot, L. (2008), Étudier les pratiques d'enseignement. Un exemple comparatif au collège et à l'école primaire. Les Dossiers des Sciences de l'Éducation, 19, p. 81-101.

Veillard, L., \& Tiberghien, A. (À paraître). Instrumentation de la recherche en Éducation. Le cas du développement d'une base de vidéos de situation d'enseignement et d'apprentissage ViSA. Paris: Maison des Sciences de l'Homme. 


\section{ANNEXES}

Extraits du programme de sciences physiques de la classe de seconde

Référence: MEN (Ministère de l'éducation nationale). (2010). Programmes d'enseignement de la classe de seconde. Bulletin officiel, 4 ( $\mathrm{n}^{\circ} 4$ du 29 avril 2010 Bulletin officiel spécial).

\section{L'entrée thématique de l'enseignement}

La prise en compte de la diversité des publics accueillis en classe de seconde nécessite une adaptation des démarches et des progressions. La présentation des programmes sous forme de thèmes a été retenue pour répondre à cette nécessité car elle offre au professeur une plus grande liberté pédagogique qu'une présentation classique pour aborder les notions de chimie et de physique.

L'approche thématique permet aussi de développer l'intérêt pour les sciences en donnant du sens aux contenus enseignés en explorant des domaines très divers, tout en gardant un fil conducteur qui assure une cohérence à l'ensemble des notions introduites. L'enseignement thématique se prête particulièrement bien à la réalisation de projets d'élèves, individualisés ou en groupes. Ces projets placent les élèves en situation d'activité intellectuelle, facilitent l'acquisition de compétences et le conduisent à devenir autonome.

Trois thèmes relatifs à la santé, la pratique sportive, et à l'Univers constituent le programme.

\section{L'univers}

L'Homme a de tout temps observé les astres afin de se situer dans l'Univers. L'analyse de la lumière émise par les étoiles lui a permis d'en connaître la composition ainsi que celle de leur atmosphère et de la matière interstellaire. L'étude du mouvement des planètes autour du Soleil l'a conduit à la loi de gravitation universelle. Il apparaît ainsi que le monde matériel présente une unité structurale fondée sur l'universalité des atomes et des lois.

\begin{tabular}{|c|c|}
\hline NOTIONS ET CONTENUS & COMPÉTENCES ATTENDUES \\
\hline
\end{tabular}

Une première présentation de l'Univers: le remplissage de l'espace par la matière est essentiellement lacunaire aussi bien au niveau de l'atome qu'à l'échelle cosmique. Les dimensions de l'Univers sont telles que la distance parcourue par la lumière en une année est l'unité adaptée à leur mesure.

\begin{tabular}{l|l}
$\begin{array}{l}\text { Description de l'Univers: l'atome, la Terre, le système solaire, la } \\
\text { Galaxie, les autres galaxies, exoplanètes et systèmes planétaires } \\
\text { extrasolaires }\end{array}$ & $\begin{array}{l}\text { Savoir que le remplissage de l'espace par la matière est essentiel- } \\
\text { lement lacunaire, aussi bien au niveau de l'atome qu'à l'échelle } \\
\text { cosmique. }\end{array}$ \\
$\begin{array}{l}\text { Propagation rectiligne de la lumière. } \\
\text { Vitesse de la lumière dans le vide et dans l'air. }\end{array}$ & $\begin{array}{l}\text { Connaître la valeur de la vitesse de la lumière dans le vide (ou } \\
\text { dans l'air). }\end{array}$ \\
L'année de lumière. & $\begin{array}{l}\text { Connaître la définition de l'année de lumière et son intérêt. } \\
\text { Expliquer l'expression: « voir loin, c'est voir dans le passé ». } \\
\text { Utiliser les puissances de 10 dans l'évaluation des ordres de } \\
\text { grandeur. }\end{array}$ \\
\hline
\end{tabular}


Les étoiles: l'analyse de la lumière provenant des étoiles donne des informations sur leur température et leur composition. Cette analyse nécessite l'utilisation de systèmes dispersifs.

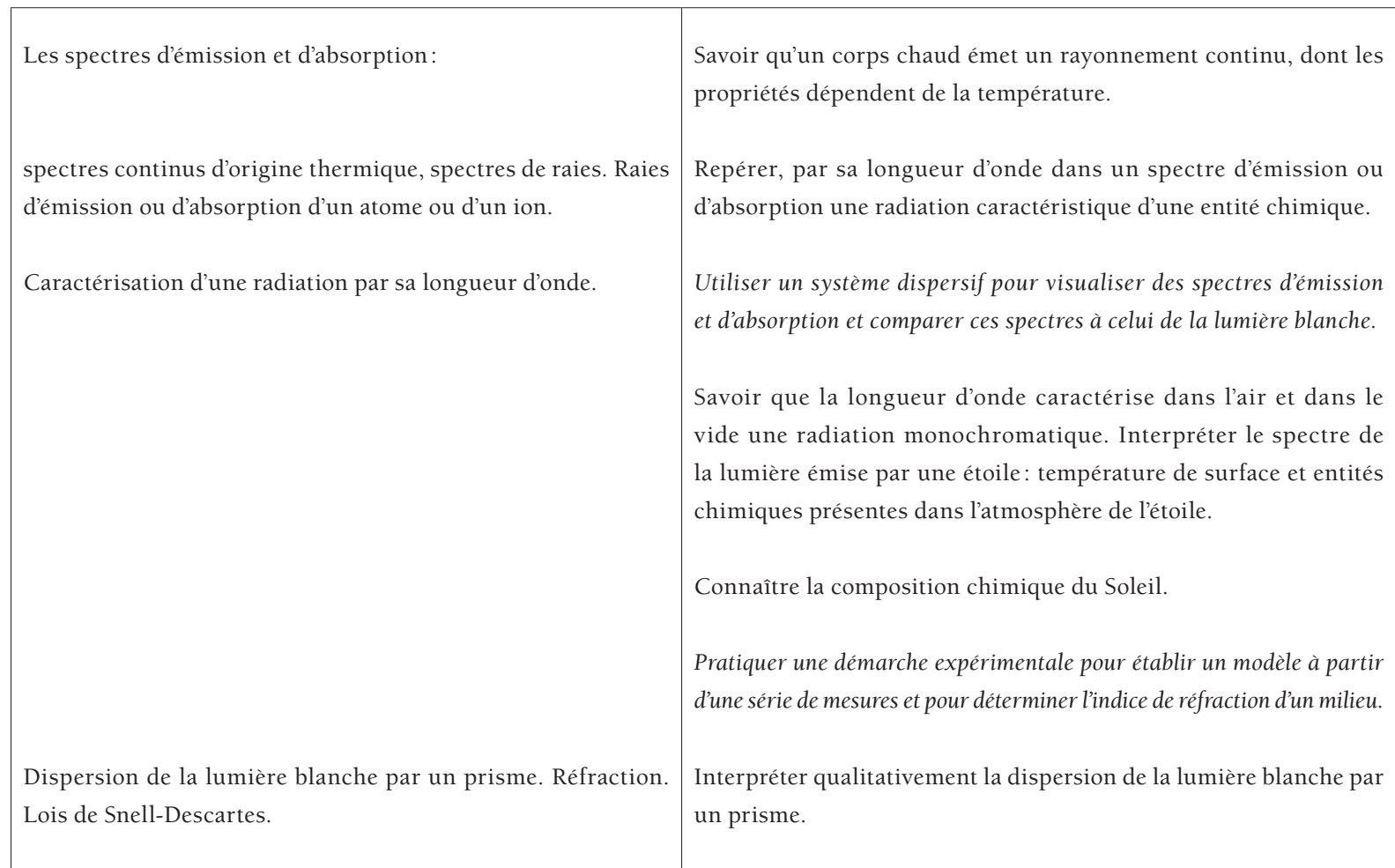

